\title{
Potentiometric study of polyaniline film synthesized with various dopants and composite-dopant: A comparative study
}

\author{
P D GAIKWAD, D J ShIRALE, V K GADE, P A SAVAle, K P KAKDE, H J KHARAT \\ and M D SHIRSAT* \\ Department of Physics, Dr B A Marathwada University, Aurangabad 431 004, India
}

MS received 20 March 2006; revised 17 May 2006

\begin{abstract}
The potentiometric study of polyaniline (PANI) film synthesized with dopants viz. polyvinyl sulfonic acid (PVS), $p$-toluene sulfonic acid ( $p$ TS), dodecyl benzene sulfonic acid (DBS) and composite-dopants viz. PVS- $p$ TS and PVS-DBS, has been carried out. The synthesized PANI films were characterized by electrochemical technique, UV-visible spectroscopy, Fourier transform infrared spectroscopy (FTIR), scanning electron microscope (SEM) and conductivity measurement. It was found that the PANI doped with PVS gives good electrochemical properties, conductivity as well as surface morphology as compared to $p$ TS and DBS, whereas in composite dopants the PANI doped with PVS- $p$ TS gives good polymer matrix as compared to PVS-DBS.
\end{abstract}

Keywords. Polyaniline; dopants; composites dopants; potentiometric.

\section{Introduction}

Amongst conducting polymers, polyaniline (PANI) receives greater attention as a conducting organic material due to its good environmental stability (Lacroin and Diaz 1988; Camalet et al 1998). Polyaniline has many advantages, which include simplicity and rapidity of preparation by electrochemical methods and the ability to be formed in aqueous electrolytic solutions (Ramanathan et al 1994). PANI is recognized to be an air-stable organic conducting polymer with interesting electrochemical properties which makes it suitable for a number of practical applications, such as in biosensors (Roth and Graupher 1993), EMI shielding (Bartlett and Birkin 1993), light weight batteries (Depaoli et al 1999), electrochromic display devices (Delccuw et al 1997), electronic devices (Kaneto et al 1998), actuators (Chen and Cho 1996), and electrochromic materials (Skotheim et al 1998).

Organic conducting polymers have recently emerged as a new class of electroactive materials and are interesting subjects for research and development (Chen and Cho 1996; Delccuw et al 1997; Kaneto et al 1998; Skotheim et al 1998; Shirale et al 2006). The remarkable switching capability of these electroactive materials between the conducting oxidized (doped) and the insulating-reduced (undoped) state is the basis of many applications. Among others, the poly-conjugated conducting polymers have been recently proposed for biosensing applications because of a number of favourable characteristics such as (i) direct

\footnotetext{
*Author for correspondence (mdshirsat_bamu@yahoo.co.in)
}

and easy deposition on sensor electrode by electrochemical oxidation of monomer, (ii) control of thickness and (iii) redox conductivity. Various conducting polymers have been extensively considered as the material for immobilization of enzymes such as polyacetylene, polythiophene, polypyrrole (Ppy), polyindole and polyaniline. Among these conducting polymers, PANI is well characterized and is probably one of the most suitable polymers for biosensor applications because it has good environmental stability and biocompatibility. Its low oxidation potential enables a conducting polymer film to be grown from aqueous solutions that is compatible with most of the biological elements. Moreover, its easy polymerization, high electrical conductivity, chemical stability and ability to form freestanding films are added advantages for its application to biosensors (Shaolin et al 1991; Ramanathan et al 1994).

The PANI film can be doped with small anion $\left(\mathrm{Cl}^{-}\right.$, $\mathrm{NO}_{3}^{-}, \mathrm{HSO}_{4}^{-}$), which can be incorporated into the film on oxidation. However, the polymer matrix forms with small anion has poor stability. To overcome this problem, the PANI film need to be synthesized with a large anion like polyelectrolyte e.g. PVS. Since the large anions are not able to leave the polymer matrix it can result into a stable polymer matrix. The polymer synthesized with PVS indicates that ions inserted into the films maintain the charge neutrality during reduction, which is very useful for the immobilization of bio-component. The presence of polyelectrolyte in the polymerization solution results in increased growth rate, higher compactness and improved environmental stability of the synthesized film (Otero and Olazabal 1996; Hallik et al 2001). The organic sulfonic acid, which is milder, proved to be a better agent for the 
formation of $\mathrm{H}$-bond. The difference in the electronics spectra may also be attributed to fewer quinoneimine moieties in the polymer synthesized in presence of organic sulfonic acid (Vachin et al 1987). Sulfonic acid group was introduced into PANI backbone by sulfonation of the emeraldine and leucoemeraldine state to get self doped PANI (Yue et al 1991; Yang and Wen 1994) in which the conductivity of PANI can be extended to neutral $\mathrm{pH}$.

In our previous article (Gaikwad et al 2006), we have optimized the electrochemical parameters of PANI film doped with small anion i.e. $\mathrm{HSO}_{4}^{-}$. The aim of the present investigation is to improve the stability of synthesized PANI film by using large and medium sized anions viz. PVS, $p$ TS, DBS and composite dopants: PVS $-p$ TS and PVS-DBS.

\section{Experimental}

The aniline was distilled twice before use. We have used the dopants, poly vinyl sulfonic acid (PVS) (130.09 MW), procured from Aldrich (25 wt\% solution in water), $p$ toluene sulfonic acid ( $p$ TS) and dodecyl benzene sulfonic acid (DBS) procured from Loba Chemie. An aqueous solution of aniline (99\%), dopant and composite dopant were prepared in double distilled water. The concentrations of aniline and supporting electrolytes were $0.2 \mathrm{M}$ and $0.5 \mathrm{M}$, respectively. The $\mathrm{pH}$ was adjusted by adding nitric acid and/or sodium hydroxide and measured by calibrated $\mathrm{pH}$ meter.

The electrochemical polymerization of aniline was carried out by potentiometric method in one compartment electrochemical cell. Platinum was used as a counter electrode (cathode) and another ITO glass was used as a working electrode (anode). The reference electrode was silver-silver chloride $(\mathrm{Ag} / \mathrm{AgCl})$. All three electrodes were placed vertically in an electrochemical cell. An $80 \mathrm{ml}$ solution was used for each reaction. The $\mathrm{pH}$ of the electrolyte was measured by calibrated ELICO LI120 pH meter. The characterization of polyaniline film was carried out by electrochemical technique using an indigenously designed and fabricated electrochemical polymerization system, UVvisible spectroscopy 1601 Shimadzu, FTIR spectroscopy by using Testscan Shimadzu FTIR-8400 series in the region between 350 and $4000 \mathrm{~cm}^{-1}$, conductivity measurement using four-probe method and scanning electron micrograph (SEM) using JEOL JSM-6360 analytical system.

\section{Results and discussion}

The potential-time curve recorded during the synthesis of PANI films with dopant PVS, $p$ TS and DBS is shown in figure 1. In case of potentiometric deposition, during first few seconds in the beginning of reaction, the potential increases suddenly probably indicating the difficult formation of dimmers and oligomers, after this the potential becomes almost constant suggesting that building up of the film proceeds according to the same reaction along the full thickness of the polymer. The PANI film was potentiometrically synthesized with $\mathrm{pH} 1$ and current density, $1 \mathrm{~mA} / \mathrm{cm}^{2}$, at room temperature $\left(27^{\circ} \mathrm{C}\right)$. It is reported that the polymerization potential should be minimum in order to have high conductivity of the polymer film (Shirale et al 2006). The lowest polymerization potential was recorded for PVS as compared with $p$ TS and DBS (figure 1). This indicates that the synthesized PANI film with PVS dopant will have higher conductivity than that of other two dopants and follows the order PVS > pTS > DBS, which may depend upon the size of anion. The large anion size of PVS causes to enhance the growth rate, compactness and ultimately the stability of polymer matrix. The $p$ TS and DBS (medium anion size) may be less stable within the polymer matrix as compared to PVS (large anion size). The conductivity of the synthesized PANI film for various dopants is shown in table 1. It shows very good agreement with polymerization potential. It clearly reveals that the synthesized PANI film with PVS will have higher conductivity and provides more stable polymerization matrix, which is an added advantage for the immobilization of biocomponent.

The potential-time curve recorded during synthesis of PANI films with dopants PVS, $p$ TS and the composite dopant PVS- $p$ TS is shown in figure 2. It can be seen that lowest polymerization potential was recorded for PVS

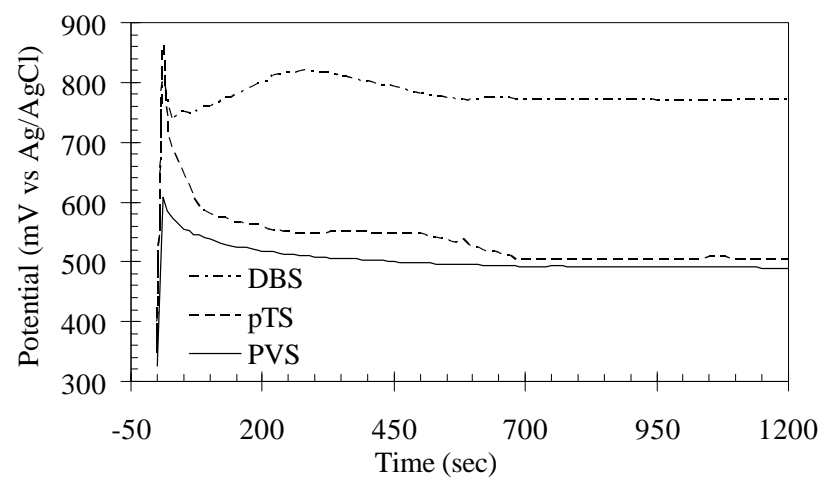

Figure 1. Potential-time curve of PANI films with dopant PVS, $p$ TS and DBS for $1.0 \mathrm{pH}, 1 \mathrm{~mA} / \mathrm{cm}^{2}$ current density and temperature, $27^{\circ} \mathrm{C}$.

Table 1. Relation between the polymerization potential and conductivity of various dopants and composite dopants.

\begin{tabular}{lccc}
\hline $\begin{array}{l}\text { S1 } \\
\text { no. }\end{array}$ & $\begin{array}{c}\text { PANI film with } \\
\text { dopants }\end{array}$ & $\begin{array}{c}\text { Polymerization } \\
\text { potential }(\mathrm{mV})\end{array}$ & $\begin{array}{c}\text { Conductivity } \\
(\mathrm{S} / \mathrm{cm})\end{array}$ \\
\hline 1 & PVS & 490 & $1 \cdot 6$ \\
2 & PVS- $p$ TS & 491 & $1 \cdot 54$ \\
3 & $p$ TS & 503 & $1 \cdot 2$ \\
4 & PVS-DBS & 508 & $1 \cdot 02$ \\
5 & DBS & 771 & $0 \cdot 51$ \\
\hline
\end{tabular}


and little high potential recorded for PVS- $p$ TS. The conductivity of synthesized PANI film with PVS $-p$ TS is $1.54 \mathrm{~S} / \mathrm{cm}$, which is slightly less than that of synthesized PANI film with PVS dopant (table 1). It means that even though $p$ TS dopant has higher polymerization potential, PVS helps to reduce the polymerization potential when combined with $p$ TS.

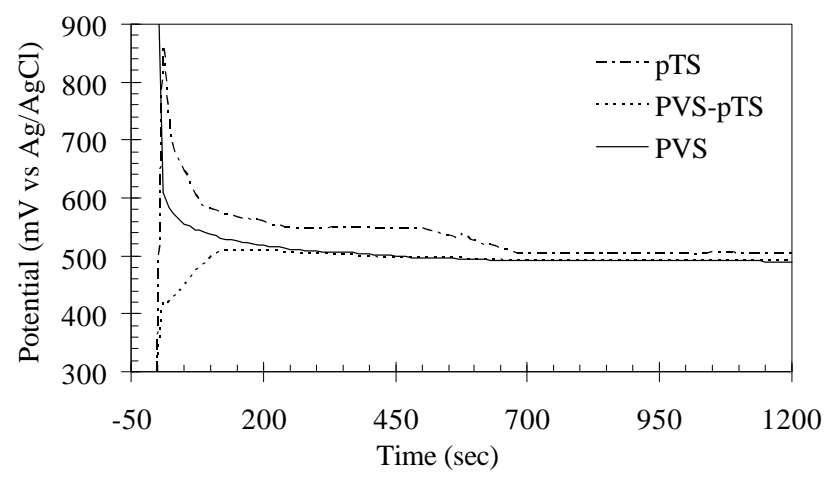

Figure 2. Potential-time curve of PANI films with dopant PVS, $p$ TS and composite dopant PVS $-p$ TS for $1.0 \mathrm{pH}, 1 \mathrm{~mA} /$ $\mathrm{cm}^{2}$ current density and temperature, $27^{\circ} \mathrm{C}$.

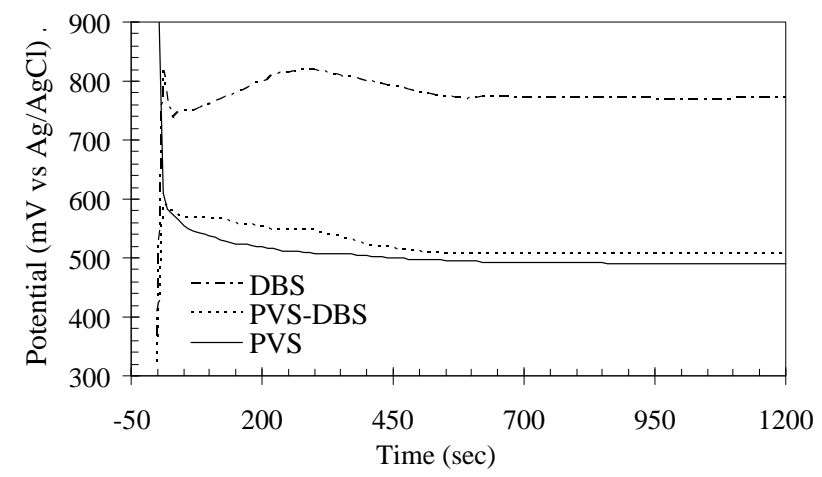

Figure 3. Potential-time curve of PANI films with dopant PVS, DBS and composite dopant, PVS-DBS, for $1.0 \mathrm{pH}$, $1 \mathrm{~mA} / \mathrm{cm}^{2}$ current density and temperature, $27^{\circ} \mathrm{C}$.

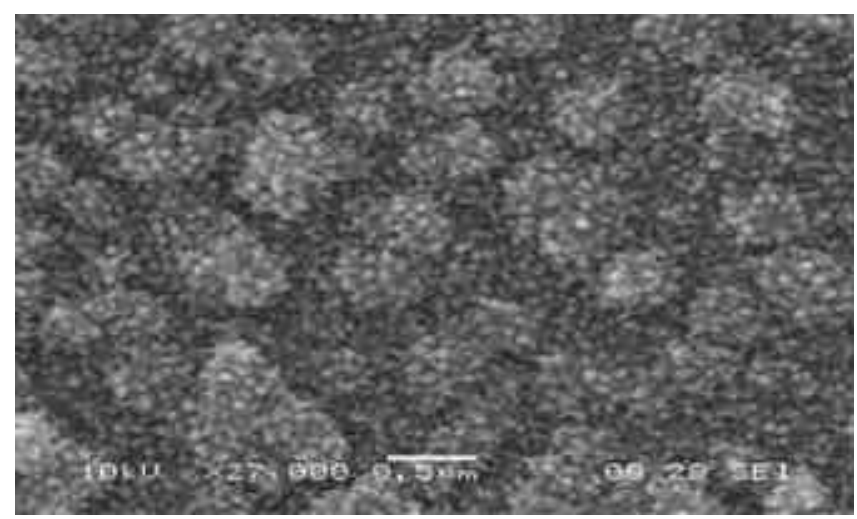

Figure 4. SEM micrograph of synthesized PANI with PVS dopant.
The potential-time curve recorded during synthesis of PANI film with dopants PVS, DBS and composite dopant PVS-DBS is shown in figure 3. The conductivity of the synthesized PANI film with composite dopant PVS-DBS is $1.02 \mathrm{~S} / \mathrm{cm}$ (table 1 ). However, if we compare the conductivity of synthesized PANI film with composite dopant, PVS- $p$ TS, with that of PVS-DBS, the former has

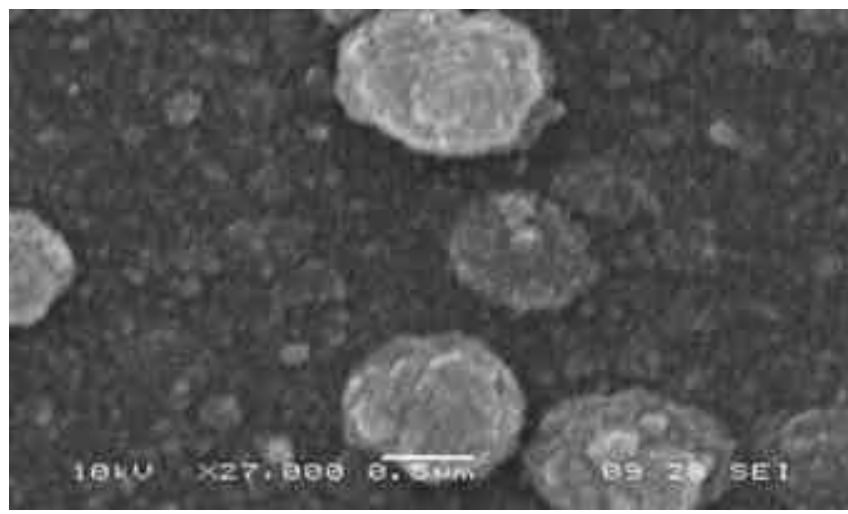

Figure 5. SEM micrograph of synthesized PANI with PVS$p$ TS composite dopant.

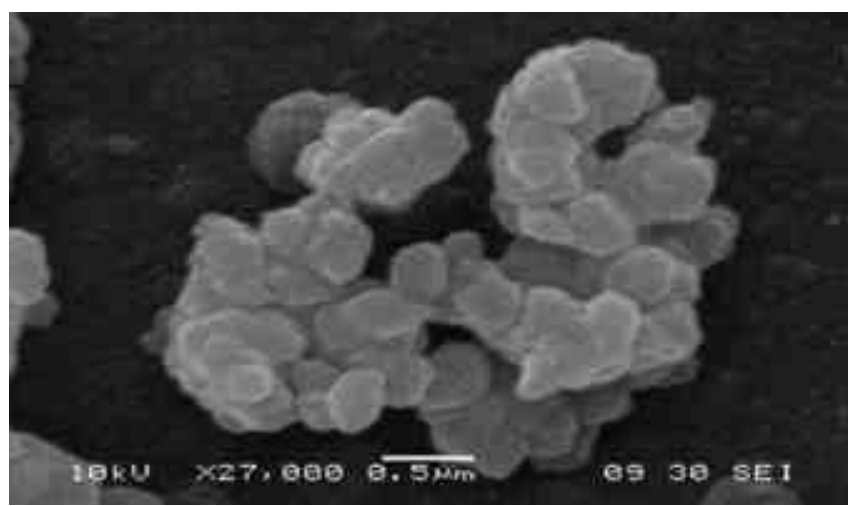

Figure 6. SEM micrograph of synthesized PANI with PVSDBS composite dopant.

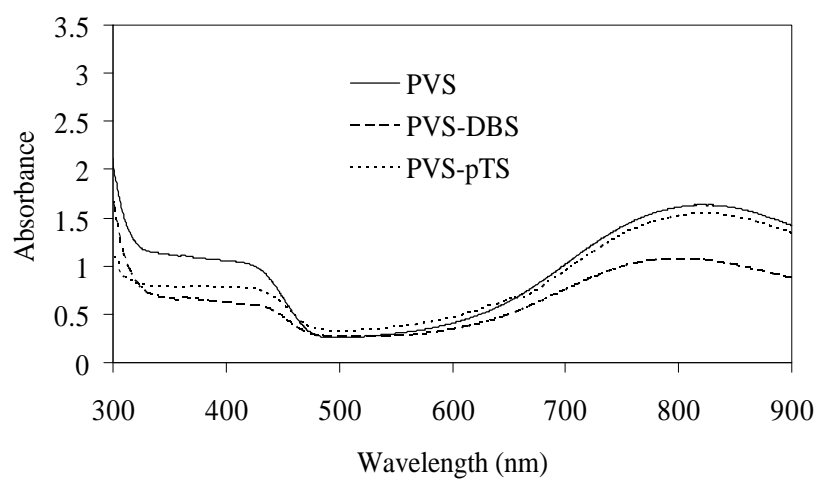

Figure 7. UV-vis spectra of synthesized PANI film with PVS, PVS- $p$ TS and PVS-DBS. 


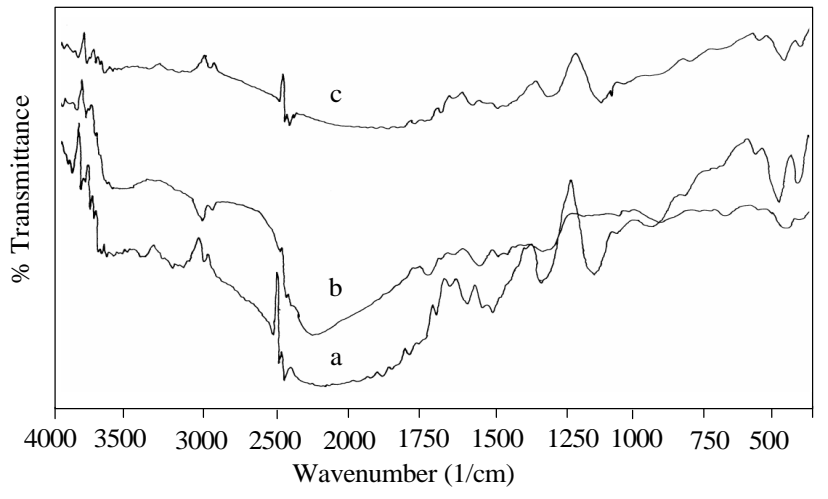

Figure 8. FTIR spectra of synthesized PANI film with a. PVS, b. PVS $-p$ TS and c. PVS-DBS.

Table 2. Relation between the absorbance and conductivity of various dopants and composite dopants.

\begin{tabular}{lccc}
\hline $\begin{array}{l}\text { Sl } \\
\text { no. }\end{array}$ & $\begin{array}{c}\text { PANI film with } \\
\text { dopants }\end{array}$ & Absorbance & $\begin{array}{c}\text { Conductivity } \\
(\text { S/cm) }\end{array}$ \\
\hline 1 & PVS & 1.61 & 1.6 \\
2 & PVS- $p$ TS & 1.53 & 1.54 \\
3 & PVS-DBS & 1.08 & 1.02 \\
\hline
\end{tabular}

higher conductivity (table 1). The synthesized PANI films with dopant, PVS and composite dopant, PVS- $-p$ TS, were uniform and adhesive. This reveals that the compositedopant, PVS-pTS, is also good for the synthesis of PANI film which can provide stable polymer matrix for the immobilization of biocomponent.

The surface morphology of PANI film synthesized with various dopants at $1 \mathrm{~mA} / \mathrm{cm}^{2}$ current density and $1 \mathrm{pH}$ was carried out by scanning electron microscope (SEM) as shown in figures 4-6 for PVS, PVS- $p$ TS and PVSDBS, respectively. The fine microspheroidal surface was observed for both PVS and composite dopant PVS- $p$ TS (figures 4 and 5). While for composite dopants (PVSDBS) (figure 6), we observed poor matrix and the polymer was not uniformly deposited over the substrate. The SEM was in good agreement with the experimental results.

UV-visible spectra of synthesized PANI film with PVS, PVS- - TS and PVS-DBS are shown in figure 7. A green coloured film showed two absorption peaks for both PVS and composite dopants, PVS $-p$ TS. The peak at $435 \mathrm{~nm}$ is because of $\pi-\pi^{*}$ transition and a broad peak around $800 \mathrm{~nm}$ corresponds to the conducting phase for both PVS and composite dopant, PVS-pTS. In case of composite dopants, PVS-DBS, we observed the peak at $783 \mathrm{~nm}$ corresponding to the conducting phase but the absorption peak is less as compared to the dopant, PVS and composite dopant, PVS $-p$ TS. The absorption spectra clearly indicate systematic increase in the overall absorbance, which is in proportion to the electrical conductivity (table 2).

The FTIR spectra of synthesized PANI film with (a) PVS, (b) PVS- $p$ TS and (c) PVS-DBS are shown in fig- ure 8 . The peak observed in the region $2000-2100 \mathrm{~cm}^{-2}$ is of typically the conducting form of the PANI film. The peak at $1533 \mathrm{~cm}^{-1}$ and $1497 \mathrm{~cm}^{-1}$ corresponds to the quinine and benzene ring stretching deformation, respectively. The $\mathrm{C}-\mathrm{N}$ stretching in the quinoid ring is observed at $1378 \mathrm{~cm}^{-1}$, while $\mathrm{C}-\mathrm{N}$ stretching of a secondary aromatic amine is observed at peak, $1038 \mathrm{~cm}^{-1}$. The peaks at 1093 and $1099 \mathrm{~cm}^{-1}$ corresponds to the $\mathrm{C}-\mathrm{H}$ in plane bending mode. $\mathrm{C}=\mathrm{N}$ stretching of quinine diimine unit is observed at $1565 \mathrm{~cm}^{-1}$. The peak observed at $1035 \mathrm{~cm}^{-1}$ and $694.3 \mathrm{~cm}^{-1}$ are due to the symmetric stretching of $\mathrm{SO}_{3}^{-}$group. Thus, the FTIR spectral results confirm the formation of polyaniline film in PVS and composite with PVS medium.

\section{Conclusions}

The polyaniline films with dopants PVS, $p$ TS, DBS and composite dopants PVS- $p$ TS, PVS-DBS have been successfully synthesized. The conductivity of the PANI film synthesized with PVS and PVS-pTS was found to be $1.6 \mathrm{~S} / \mathrm{cm}$ and $1.54 \mathrm{~S} / \mathrm{cm}$, respectively. The UV-visible spectra show systematic changes with conductivity. The FTIR spectra confirm the formation of PANI in the presence of PVS and PVS- $p$ TS. The SEM confirms the uniformity of synthesized PANI film with PVS and PVS $-p$ TS.

\section{Acknowledgements}

Authors are thankful to the University Grants Commission, New Delhi, for financial assistance. They also thank the Departments of Chemistry and Physics, University of Pune, Pune, for extending FTIR and SEM facilities.

\section{References}

Bartlett P N and Birkin P R 1993 Anal. Chem. 651118

Camalet O C et al 1998 Synth. Met. 93133

Chen S A and Cho C J 1996 Synth. Met. 7993

Delccuw O M et al 1997 Synth. Met. 8753

Depaoli M A et al 1999 Electrochim. Acta 442983

Gaikwad P D et al 2006 Bull. Mater. Sci. 29169

Hallik A et al 2001 J. Solid State Electrochem. 5265

Kaneto K et al 1998 Synth. Met. 712211

Lacroin J C and Diaz A F 1988 J. Electrochem. Soc. E135 1457

Otero T F and Olazabal V 1996 Electrochim. Acta 41213

Ramanathan K, Annapoorni S and Malhotra B D 1994 Sens. Actuat. 21165

Roth S and Graupher W 1993 Synth. Met. 573623

Shaolin M, Huaiguo X and Bidong Q J 1991 Electroanal. Chem. 3047

Shirale D J et al 2006 Mater. Lett. 601407

Skotheim T A, Elsenbaumer R L and Reynolds J R 1998 Handbook of conducting polymers (New York: Marcel Dekker) 2nd ed.

Vachin D et al 1987 Synth. Met. 18297

Yang C N and Wen T C 1994 J. Appl. Electrochem. 24166

Yue J W et al 1991 J. Am. Chem. Soc. 1132265 